\title{
Influence of subunit structure on the oligomerization state of light harvesting complexes: a free energy calculation study
}

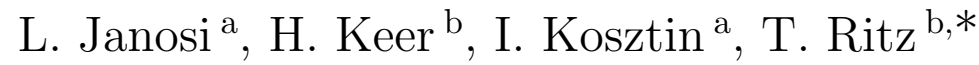 \\ ${ }^{a}$ Department of Physics and Astronomy, University of Missouri, Columbia, MO \\ 65211, USA \\ ${ }^{\mathrm{b}}$ Department of Physics and Astronomy, University of California, Irvine, CA \\ 92697, USA
}

\begin{abstract}
Light harvesting complexes 2 (LH2) from Rhodospirillum (Rs.) molischianum and Rhodopseudomonas (Rps.) acidophila form ring complexes out of eight or nine identical subunits, respectively. Here, we investigate computationally what factors govern the different ring sizes. Starting from the crystal structure geometries, we embed two subunits of each species into their native lipid-bilayer/water environment. Using molecular dynamics simulations with umbrella sampling and steered molecular dynamics, we probe the free energy profiles along two reaction coordinates, the angle and the distance between two subunits. We find that two subunits prefer to arrange at distinctly different angles, depending on the species, at about $42.5^{\circ}$ for $R$ s. molischianum and at about $38.5^{\circ}$ for Rps. acidophila, which is likely to be an important factor contributing to the assembly into different ring sizes. Our calculations suggest a key role of surface contacts within the transmembrane domain in constraining these angles, whereas the strongest interactions stabilizing the subunit dimers are found in the C-, and to a lesser extent, N-terminal domains. The presented computational approach provides a promising starting point to investigate the factors contributing to the assembly of protein complexes, in particular if combined with modeling of genetic variants.
\end{abstract}

Key words: membrane protein, transmembrane helix, protein complex assembly, bacteriochlorophyll protein

* Corresponding author. Tel.: ++1-949-824-4345

Email addresses: KosztinI@missouri.edu (I. Kosztin), tritz@uci.edu

(T. Ritz).

Preprint submitted to Elsevier Science

23 March, 2005 


\section{Introduction}

In photosynthesis, assemblies of pigment-protein complexes absorb sunlight and convert its energy into a biochemical potential. In recent years, tremendous progress has been made towards identifying the in vivo structure of the photosynthetic apparatus, in particular that of purple bacteria [1-4]. Calculations and spectroscopic measurements reveal that a close relationship exists between the efficiency of light harvesting and the geometrical arrangements of pigment-protein complexes [5-8].

This raises the question as to how nature governs the assembly of its photosynthetic apparatus within its membrane. The antenna light-harvesting complex 2 (LH2) of purple bacteria can be considered a paradigmatic model system to address this question, because (i) atomic-resolution crystal structures exist for LH2s with different organizations and (ii) mutagenesis and reconstitution assays allow for direct experimental studies of key factors in the assembly of LH2 complexes.

LH2s are composed of repetitions of a basic unit of two transmembrane polypeptides, $\alpha$ and $\beta$. Each $\alpha \beta$ heterodimeric subunit non-covalently binds three bacteriochlorophylls (BChls) and one carotenoid.Figure 1 shows the basic subunit of LH2 from Rhodospirillum (Rs.) molischianum and Rhodopseudomonas (Rps.) acidophila, respectively, as taken from their crystal structures $[9,10]$. Figure 2 shows the corresponding sequences of LH2 $\alpha$ and $\beta$ polypeptides. Both $\alpha$ and $\beta$ polypeptides consist of polar $\mathrm{N}$ - and C-termini and a central hydrophobic region. The N-termini lie on the cytoplasmic side of the membrane, the C-termini on the periplasmic side. Amino acids in the central hydrophobic region form two transmembrane $\alpha$-helices. B850 BChls are ligated to the highly conserved $\mathrm{His}_{0}$ residues near the C-terminus.

Interestingly, the crystallographic structures of LH2 reveal a different organization of subunits, a ring of nine $\alpha \beta$-subunits for Rps. acidophila $[9,11]$, but of eight $\alpha \beta$-subunits for Rs. molischianum [10]. EM and AFM studies reveal nonameric organizations for LH2s from Rhodovulum sufidophilum [12], Rhodobacter (Rb.) sphaeroides [13,14], and Rubrivivax gelatinosus $[15,16]$, whereas a low-light variant form of LH2 from Rps. palustris reveals an eight-fold symmetry [17]. In all of the above cases, the octameric or nonameric organization appears to be homogeneous within a given species, although chromatographies for 3D crystallization of LH2 from Rs. molischianum suggest the possibility of unstable LH2 forms with different numbers of subunits (H. Michel, personal comm.). Structural heterogeneity within a species has been described only for Rs. photometricum, where AFM studies suggest that most LH2s are organized in either eight-, nine-, or ten-fold symmetry [18]. 

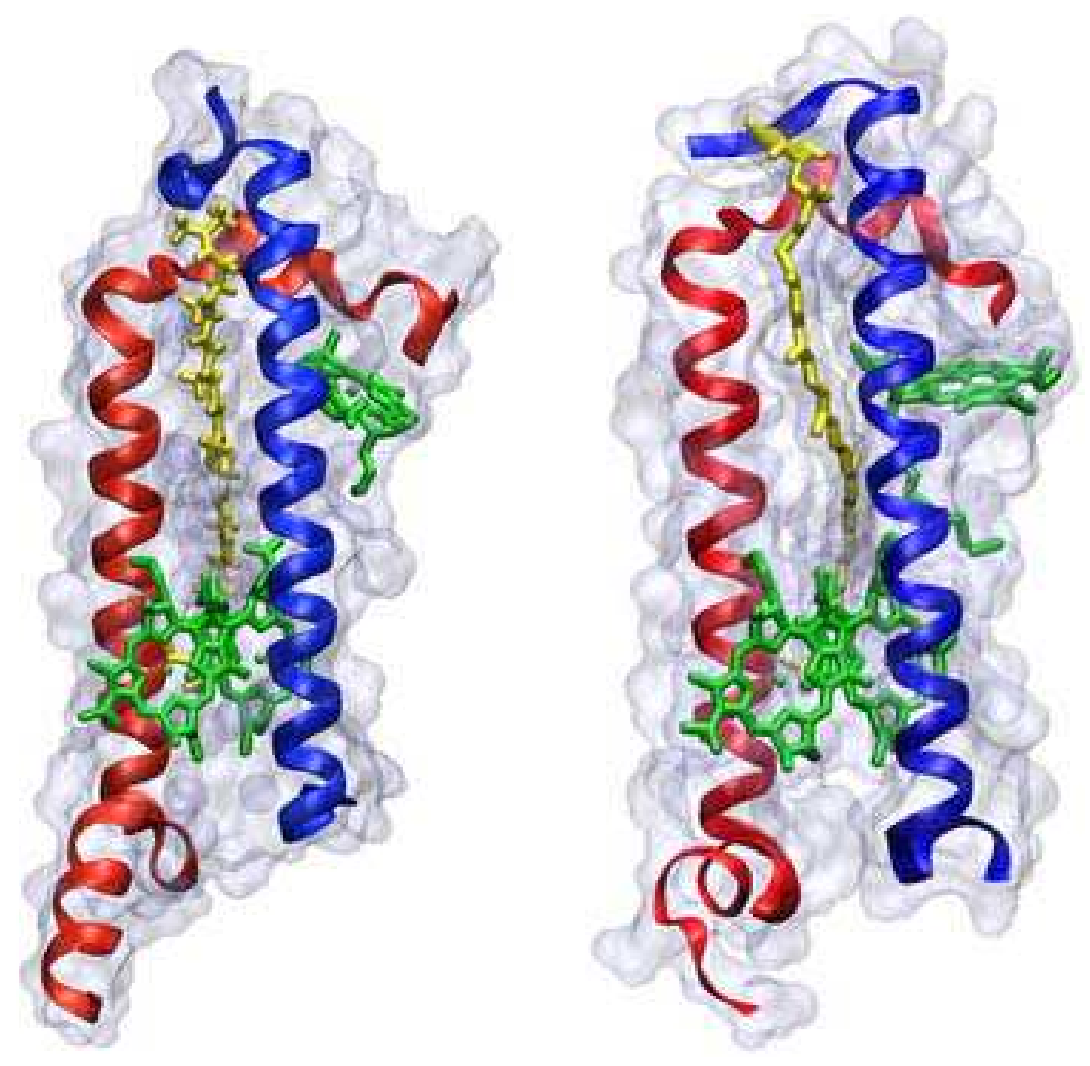

Fig. 1. Structure of a subunit of LH2 from Rs. molischianum (left) and from Rps. acidophila (right). Each subunit consists of one $\alpha$ polypeptide (blue ribbon), one $\beta$-polypeptide (red ribbon), three BChls (green; phytyl chains truncated), and one carotenoid (yellow). The N-terminal domains are on top, the C-terminal domains on bottom. The surface of the subunit is superimposed onto the simplified representations

LH2 $\alpha$

Rps. acidophila

MNQGKIWTVVNPAIGIPALLPSVTVIAILVHLAILSHTTWFPAYWQGGVKK

Rs. molischianum

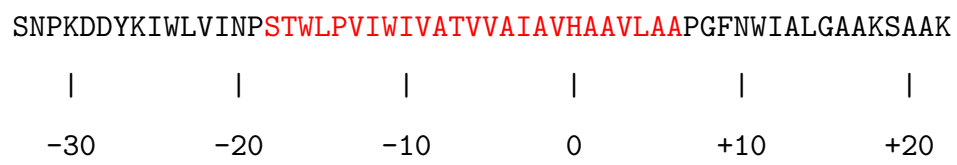

LH2 $\beta$

Rps. acidophila

ATLTAEQSEELHKYVIDGTRVFLGLALVAHFLAFSATPWLH

Rs. molischianum

AERSLSGLTEEEAIAVHDQFKTTFSAFIILAAVAHVLVWVWKPWF

$\begin{array}{ccccc}1 & 1 & 1 & 1 & 1 \\ -30 & -20 & -10 & 0 & +10\end{array}$

Fig. 2. Amino acid sequences of the light-harvesting polypeptides of LH2 from Rs. molischianum and Rps. acidophila. Transmembrane helical domains are highlighted in red. 
Differences in organization have also been reported for the core light-harvesting complex 1 (LH1), surrounding the photosynthetic reaction center. LH1, like LH2, is composed of repetitions of an $\alpha \beta$ heterodimeric subunit; however it contains only two instead of three BChls per subunit. LH1 complexes were reported to be formed of $16 \alpha \beta$-subunits (Rsp. rubrum [19], Blastochloris viridis [20]), $15 \alpha \beta$-subunits and one PufX-like peptide Rps. palustris [21], or $12 \alpha \beta$ subunits plus one PufX peptide plus a gap (Rb. sphaeroides [3,22])

Reconstitution assays [23] show that in many cases light harvesting complexes with very similar optical properties to the wild-type complexes can be reconstituted in vitro from their individual components [24,25]. Truncated versions of natural proteins, chemically synthesized de novo proteins, and mutagenetic gene products have been studied, revealing residues essential to formation of $\alpha \beta$-subunits and full complexes [26-30]. A recent study demonstrated in vivo assembly of redesigned peptides from $R b$. sphaeroides into fully functional light-harvesting complexes [31]

These results suggest that the building blocks of light harvesting complexes can self-assemble to form stable, functional complexes. Thus, one should be able to relate the observed differences in complex organization to the structure of their subunits. What features of the subunits govern the organization of the complete ring complexes, in particular the oligomerization state, i.e., the number of subunits employed in forming a ring?

In the present manuscript, we investigate in how far the variations in oligomerization states can be explained by changes in the local interaction angle between neighboring subunits. In theory, one expects that subunits with a preferred interaction angle of $45^{\circ}$ would assemble into a ring of eight subunits $\left(8 \times 45^{\circ}=360^{\circ}\right)$, whereas subunits with a preferred interaction angle of $40^{\circ}$ should form rings of nine subunits. It appears a remarkable feat for subunits of two helical proteins to control a difference in angle as small as $5^{\circ}$ in the presence of conformational fluctuations and solvent dynamics.

Starting from the crystal structures of LH2 from Rs. molischianum and Rps. acidophila, henceforth referred to as MOLI and ACI, respectively, we embed two $\alpha \beta$-subunits from either species into their native lipid-water environment. We use molecular dynamics with umbrella sampling and steered molecular dynamics to probe the free energy surfaces along various reaction coordinates, namely changes in the angle and distance between the subunits. The calculation techniques, reviewed in the following section, represent, arguably, the most accurate calculations possible on a system of this scale. They provide a reference point against which results from simpler models can be compared. The calculations reveal significant differences between subunits from MOLI and ACI that we discuss with view of the consequences for protein complex assembly. We discuss future studies necessary to capture the essential proper- 
ties governing the oligomerization state of light harvesting complexes.

\section{Theoretical and Computational Model}

As a first step in understanding how LH2 subunits assemble into precise ring structures, we have focused on determining and characterizing the interaction between two LH2 subunits. We started from a geometry characteristic to a full aggregate. Atomic models for two LH2 subunit dimers, namely for MOLI and ACI, can be readily built starting from their high resolution crystal structures available from the Protein Data Bank (entry codes 1LGH [32] and 1KZU [33], respectively). In order to mimic their native environment, we have embedded a pair of subunits for both MOLI and ACI into properly solvated lipid bilayers. The dynamical behavior of the systems were investigated by means of all atom molecular dynamics (MD) simulations. Details about the built systems and the MD protocols used are presented at the end of this section.

Since, presently, MD simulations studies are restricted to the 10-100 nanosecond time scale, they cannot be applied directly to describe the complete assembly process between two LH2 subunits. Indeed, lateral diffusion of these protein units in the lipid membrane occurs on a much longer time scale than the one accessible by MD simulations. However, suitably designed MD simulations, combined with statistical mechanical analysis, can be used to determine the free energy profile or potential of mean force (PMF) [34] between the interacting subunits. The PMF then can be used as input in a suitable stochastic model (e.g., Fokker-Planck or Smoluchowski equation [35]) for describing the dynamics of the system at a mesoscopic or even macroscopic scale. To this end, one first needs to identify a small number of variables (e.g., distances and/or angles), hereafter referred to as reaction coordinates that describe the relative separation and orientation of the subunits. Then, one should use a properly designed set of equilibrium or non-equilibrium MD simulations to calculate the PMF (i.e, free energy) $U(Q)$ of the system as a function of the reaction coordinates $Q$. Once the PMF is determined, the generalized force exerted between the subunits is equal to $F=-\partial U(Q) / \partial Q$.

In what follows, we define reaction coordinates suitable for describing the self-assembly of LH2 subunits. Then we briefly describe two well established methods for calculating PMFs from MD simulations, namely (i) umbrella sampling [36,37] combined with the weighted histogram analysis method (WHAM) [38], and (ii) steered molecular dynamics (SMD) [39,40] in conjunction with the application of the Jarzynski equality [41]. Method (i) uses equilibrium MD simulations, while (ii) relies on non-equilibrium simulations [34]. 


\subsection{Reaction Coordinates $R$ and $\theta$}

The self-assembly of LH2 rings can be envisioned as a process in which preformed LH2 subunits are first inserted into the membrane, then brought within contact distance through diffusion in the lipid membrane, followed by final locking into the ring specific geometry. In a first approximation, this process can be modeled as a purely two dimensional one in which each subunit has a specific anisotropic 2D structure (e.g., a deformed disk) that at the end of the process forms an $\mathrm{N}$-fold symmetric ring ( $\mathrm{N}=8$ for MOLI and $\mathrm{N}=9$ for $\mathrm{ACI}$ ).

We define two reaction coordinates to characterize the spatial interaction between LH2 subunits in their native membrane environment, namely $R$ - the separation between them, and $\theta$ - their relative angular orientation. A more precise definition of reaction coordinates is illustrated in Fig. 3, displaying two MOLI subunits (from the equilibrated system) in a side view (a) and in a top view (b from the N-terminal or cytoplasmic side. Fig. 3c shows only the transmembrane helical domains of the two subunits $\left(A_{1}, B_{1}\right)$ and $\left(A_{2}, B_{2}\right)$, showing a clear separation of $\alpha$ and $\beta$ polypeptides.

We define the center-of-mass (COM) of the $\alpha(\beta)$ apoproteins in subunit $i=$ 1,2 as $A_{i}\left(B_{i}\right)$. Then $\theta$ by definition is the angle made by the projections of the vectors $\mathbf{v}_{\mathbf{i}}=\overrightarrow{A_{i} B_{i}}, i=1,2$, on the $x y$-plane of the membrane. The separation distance reaction coordinate is defined as the distance between the COMs of the $\alpha \beta$-apoprotein heterodimer within the $x y$-plane, i.e., $R=\left|\mathbf{R}_{\mathbf{1}}-\mathbf{R}_{\mathbf{2}}\right|$, where $\mathbf{R}_{\mathbf{i}}, i=1,2$ are the projections of the position vectors of these COMs on the $x y$-plane. Note that, at any instant of time, the reaction coordinates are determined (through the COMs) by the coordinates and masses of a selected group of atoms from both subunits.

In selecting the group of atoms that define the reaction coordinates one has to make sure that under equilibrium (or stationary) conditions, the reaction coordinates have well defined mean values and sufficiently small fluctuations. Otherwise, the reaction coordinates are ill defined and cannot be used to characterize the system quantitatively. For example, we have found that (see Sec. 3) $R$ and $\theta$ are well defined if one considers only the heavy atoms in the transmembrane helical (TMH) domains of the $\alpha \beta$-apoproteins. Adding to the selection all the heavy atoms in the $\mathrm{N}$ - and C-terminal domains, would lead to substantial increase in the fluctuations of $\theta$ rendering the latter reaction coordinate meaningless. Finally, in what follows, we use the convention that $R$ and $\theta$ refer to particular target values of the reaction coordinates, while $\tilde{R} \equiv \tilde{R}(\mathbf{q})$ and $\tilde{\theta} \equiv \tilde{\theta}(\mathbf{q})$ refer to the instantaneous values of the reaction coordinates determined from the corresponding positions $\mathbf{q} \equiv \mathbf{q}_{j}(t)$ of all defining atoms. 


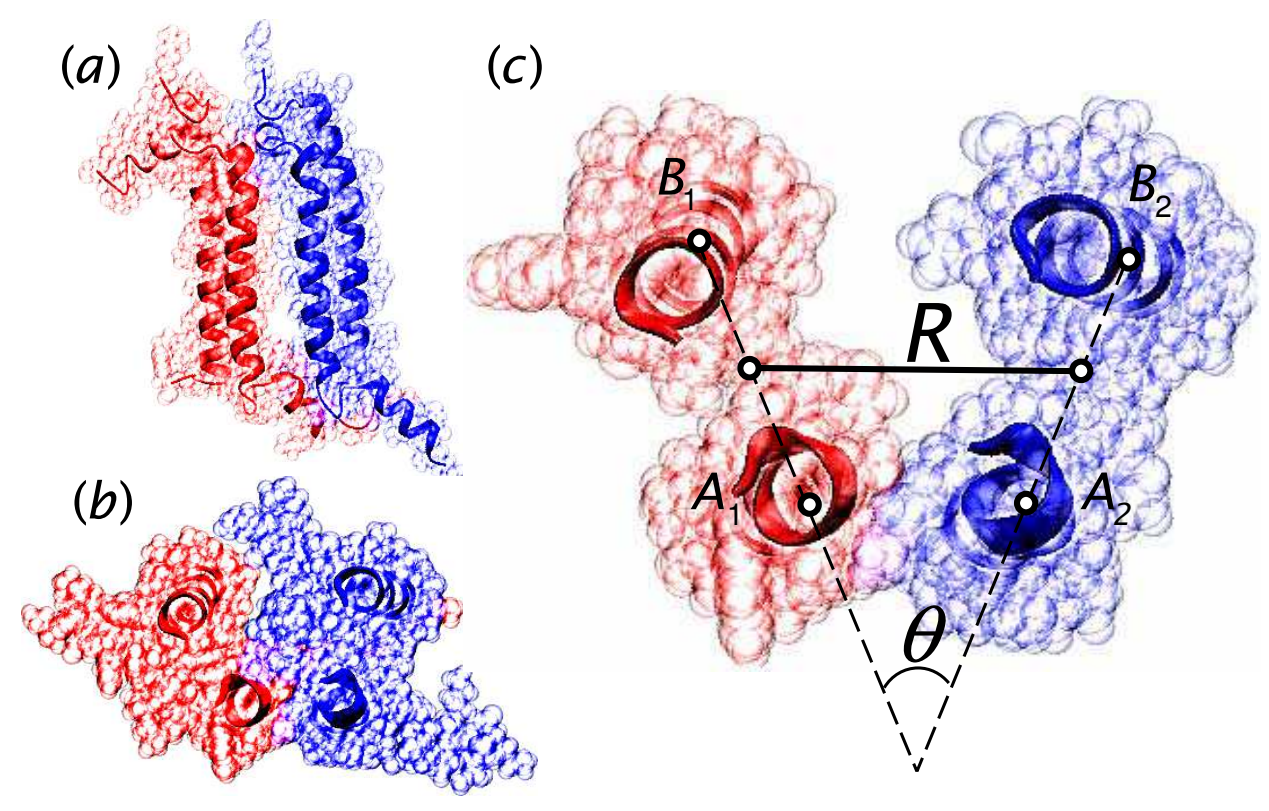

Fig. 3. Definition of the reaction coordinates $\theta$ and $R$ illustrated for two subunits of LH2 from Rs. molischianum. (a) Side view with the N-termini on top. (b) Top view from the N-terminal or cytoplasmic side in space-filling representation with transmembrane helices highlighted in ribbon representation. (c) Top view from from the N-terminal or cytoplasmic side of only the $\alpha$ and $\beta$ polypeptides with definition of reaction coordinates.

\subsection{Potential of Mean Force $U(\theta, R)$}

The PMF $U(Q) \equiv U(\theta, R)$ is the free energy of the system formed by the two interacting subunits for well defined spatial $R$ and angular $\theta$ separations; for brevity we have introduced the compact notation $Q \equiv\{\theta, R\}$. In principle, the PMF can be calculated by integrating out all the degrees of freedom except for the reaction coordinates [34], i.e.,

$$
e^{-\beta U(Q)} \equiv p_{0}(Q)=\int d \Gamma \frac{e^{-\beta H_{0}(\Gamma)}}{Z_{0}} \delta[Q-\tilde{Q}(\Gamma)],
$$

where $\beta=1 / k_{B} T$ is the temperature factor $\left(k_{B}\right.$ is the Boltzmann constant and $T$ is the absolute temperature), $p_{0}(Q)$ is the equilibrium distribution function of the reaction coordinates, $H_{0}(\Gamma)$ is the Hamiltonian of the system as a function of $\Gamma \equiv\{\mathbf{q}, \mathbf{p}\}$ (i.e., all the atomic coordinates and momenta), $Z_{0}$ is the partition function and $\delta(x)$ is the Dirac-delta function whose filtering property guarantees that the integrand in Eq. (1) is nonzero only when the reaction coordinates have the requested value, i.e, when $\tilde{Q}(\Gamma)=Q$. In principle, the equilibrium distribution function $p_{0}(Q)$ can be easily computed from MD simulations, since it is proportional to the binned histogram of the reaction coordinates calculated along the MD trajectory. Thus, the PMF is readily 
given by

$$
U(Q)=-k_{B} T \log p_{0}(Q)
$$

However, in practice, even the longest equilibrium MD trajectories will sample only a restricted region of the reaction coordinate domain (i.e., within the vicinity of the PMF minimum) of interest and the direct application of Eq. (1) is impractical.

There exist two basic methods for calculating PMFs from MD simulations widely known as (1) umbrella sampling [36], and (2) steered molecular dynamics $[42,43]$. Next we present briefly both methods within a unifying conceptual framework, and point out under what conditions one should opt for using one or the other. In both methods a crucial step is to alter the dynamics of the system by applying a suitable guiding potential.

\subsection{Harmonic Guiding Potential}

During equilibrium MD simulations the system explores only a small region of the phase space $\Gamma$ about the minimum of the sought PMF $U(Q)$. In order to properly sample energetically more difficult to reach regions, one needs to guide or steer our system towards those regions by employing, e.g., a harmonic guiding potential (HGP)

$$
V_{Q}(\tilde{Q}) \equiv V(\tilde{Q}(\Gamma) \mid Q)=\frac{k_{Q}}{2}[\tilde{Q}(\Gamma)-Q]^{2}
$$

where $k_{Q}$ is the stiffness of the HGP. With this extra potential energy, the Hamiltonian of the new, biased system becomes

$$
H_{Q}=H_{0}+V_{Q}(\tilde{Q})
$$

and, as a result, the atoms in the selections that define the reaction coordinates will experience extra forces

$$
\mathbf{F}_{i}=-\frac{\partial V_{Q}}{\partial \mathbf{r}_{i}}=-k_{Q}[\tilde{Q}(\Gamma)-Q] \frac{\partial \tilde{Q}(\Gamma)}{\partial \mathbf{r}_{i}}
$$

Thus, the HGP (3) will force the system to evolve in the phase space in such a way that during its time evolution $\tilde{Q}$ will stay confined in the vicinity of the target $Q$ value. 


\subsection{Method I: Umbrella sampling and WHAM}

In umbrella sampling, one divides the reaction coordinate interval of interest $\left(Q_{\min }, Q_{\max }\right)$ that one wants to sample in $N_{w}$ windows by conveniently chosen values $Q_{i}, i=1, \ldots, N_{w}$. Next, the reaction coordinate is sampled in each window separately by preparing identical replicas of the system and applying the harmonic guiding potential $V_{Q_{i}}(\tilde{Q})$. As a result, the biased distribution functions can be readily obtained by direct sampling of the reaction coordinate for the biased system $[34,36,38]$, i.e,

$$
p_{i}(Q)=\int d \Gamma \frac{e^{-\beta H_{i}(\Gamma}}{Z_{i}} \delta[Q-\tilde{Q}(\Gamma)]=\frac{Z_{0}}{Z_{i}} e^{-\beta V_{i}(Q)} p_{0}(Q),
$$

where, for brevity, the index $Q_{i}$ has been replaced by $i$. The equilibrium distribution in each window is related to the biased distribution of the reaction coordinate through

$$
p_{0}(Q)=\frac{Z_{i}}{Z_{0}} e^{\beta V_{i}(Q)} p_{i}(Q) .
$$

The standard method to efficiently stitch together the biased $p_{i}(Q)$ 's in order to obtain the equilibrium $p_{0}$, and therefore the sought PMF (2), is the so called weighted histogram analysis method or WHAM. According to this method, $p_{0}(Q)$ is expressed as a weighted sum over the biased distributions $p_{i}(Q)$ as follows

$$
p_{0}(Q)=\frac{\sum_{i=1}^{N_{w}} N_{i} p_{i}(Q)}{\sum_{i=1}^{N_{w}} N_{i} \frac{e^{-\beta V_{i}(Q)}}{\left\langle e^{-\beta V_{i}}\right\rangle}}
$$

where

$$
\left\langle e^{-\beta V_{i}}\right\rangle=\int d Q p_{0}(Q) e^{-\beta V_{i}(Q)} .
$$

The above non-linear coupled WHAM equations, that need to be solved iteratively, minimize the errors in determining $p_{0}(Q)$, and therefore the PMF $U(Q)$. This method will give good PMFs only if the overlap between windows is substantial. Whenever a reasonable number of well overlapping windows can be constructed, the umbrella sampling method with WHAM should be the top choice for calculating PMFs. In general, this method works very well for determining both 1D and 2D PMFs. We have used this method to calculate the $\theta$ dependent PMF for a constrained separation distance $R$ between LH2 subunits as reported in Sec. 3. 


\subsection{Method II: Steered Molecular Dynamics and Jarzynski Equality}

In many cases, it is desirable to vary in time the target value of the $\mathrm{RC}$ according to a prescribed rule. For example, we can guide or steer our system in the direction of increasing the separation distance $R$ between the two LH2 subunits during a given simulation time $\tau$ from an initial value $R_{i}$ to a final one $R_{f}$ by setting in the HGP (3)

$$
Q(t) \equiv R(t)=R_{i}+v_{R} t, \quad v_{R}=\left(R_{f}-R_{i}\right) / \tau .
$$

By choosing two or more reaction coordinates, one can easily devise complicated reaction coordinate paths along which the system can be steered using a moving HGP. With such HGP the Hamiltonian of the system becomes time dependent, and it can be expressed by inserting Eq. (10) into Eqs. (4) and (3). Then, the extra forces that need to be applied to the atoms defining the reaction coordinates can be calculated with the same formula (5). Clearly, the corresponding SMD simulations are non-equilibrium. Already a few SMD pulling simulations may help us gain some qualitative insight into the behavior of the system as it evolves along the reaction coordinate. We apply this method to investigate the correlation between $\theta$ and $R$ in LH2 subunit dimers when one of the reaction coordinates is either increased or decreased in time. The reconstruction of the PMF $U(Q)$ from such non-equilibrium simulations requires a sufficiently large number of SMD trajectories. These trajectories then need to be analyzed by employing the Jarzynski's equality that connects the free energy differences of two equilibrium states with the exponential average of the irreversible mechanical work done in non-equilibrium processes that connect the equilibrium states in question [41-44], i.e.,

$$
e^{-\beta\left(F_{Q}-F_{0}\right)}=\left\langle e^{-\beta W_{Q}}\right\rangle,
$$

where the irreversible work along a path that connects states with given $\mathrm{RC}$ values $Q_{0}$ and $Q$ is given by

$$
W_{Q}=\int_{0}^{\tau} d t \frac{d Q}{d t}\left(\frac{\partial H_{Q}}{\partial Q}\right) .
$$

Here, $\langle\ldots\rangle$ denotes the average over an ensemble of trajectories.

It should be noted that for very large switching times $\tau$ (adiabatic approximation), the work becomes reversible and we recover the expected equilibrium result $W_{\tau \rightarrow \infty}=F_{Q}-F_{0}$. On the other hand, for an instantaneous switching time $W_{\tau \rightarrow 0}=H_{Q}[\Gamma(0)]-H_{0}[\Gamma(0)]=\Delta H$, and the Jarzynski equality (11) yields again the expected result, i.e., $\exp (-\beta \Delta F)=\langle\exp (\beta \Delta H)\rangle$. 
In general, the PMF calculation based on the application of the Jarzynski equality to trajectories from SMD simulations is preferred to the umbrella sampling method whenever the fluctuations of the reaction coordinate are small and a huge number of sampling windows would be required for assuring proper overlap of the reaction coordinate distribution histograms. However, it is not totally clear how many SMD trajectories one should use in such a case for calculating the PMF with sufficient accuracy. Since for our LH2 subunit dimer system the fluctuations in $R$ are rather small, i.e., $\sim 0.2 \AA$, and a complete detachment of the subunits requires an increase in $R$ of $20-30 \AA$, the present method would be more suitable to calculate $U(R \mid \theta)$ than the umbrella sampling method.

\subsection{System modeling and MD simulations}

Here we provide a brief description of the modeling of our MOLI and ACI dimers, and of the MD simulations performed.

ACI dimer - two adjacent complete LH2 subunits (protein and cofactors) were extracted from the PDB structure 1KZU [33]. After adding the missing hydrogens, the protein complex was inserted in a pre-equilibrated and hydrated POPC lipid bilayer. Finally, the system was solvated by adding extra water layers to the two sides of the lipid bilayer. Besides the proteins and cofactors the system contained 4014 water molecules and 169 POPC lipid molecules, with a total system size of 38,594 atoms. The +4 e charge of the system was neutralized by properly adding $4 \mathrm{Cl}^{-}$counter ions. The system was built by using XPLOR [45] and VMD [46].

MOLI dimer - using VMD [46] and its plugins, two complete neighboring units extracted from a fully equilibrated 8-fold LH2 MOLI ring, reported in one of our previous MD studies [47] were inserted in a pre-equilibrated and hydrated POPE lipid bilayer, and then solvated by adding two pre-equilibrated $8 \AA$ thick water layers to each side of the membrane. The $+4 \mathrm{e}$ charge of the system was neutralized by properly adding $4 \mathrm{Cl}^{-}$counter ions. In addition to the protein and cofactors, the final system contained 8299 water molecules and 128 POPE lipids, with a total system size of 44,997 atoms.

Equilibrium MD simulations - After proper energy minimization, the ACI (MOLI) system was equilibrated at $300 \mathrm{~K}(310 \mathrm{~K})$, normal atmospheric pressure through a $4 \mathrm{~ns}$ ( $5 \mathrm{~ns}$ ) long MD simulation in the NPT ensemble. Periodic boundary conditions and full electrostatics via the Particle Mash Ewald method were used. All MD simulations were carried out with the NAMD [48] program using the CHARMM 27 parameter set [49]. The simulations were carried out on local Linux Beowulf clusters, and the required time for $1 \mathrm{~ns}$ 
simulation running on 30 CPUs was about 1.5 days.

SMD simulations - A total of 20 SMD simulations were carried out for each system, starting from the same state that coincided with the last frame of equilibrium MD run. The details of the applied harmonic guiding potential for each of the runs are described in Sec. 3.2.

Umbrella sampling simulations - To determine the PMF $U(\theta \mid R)$ for $R=R_{0}=$ $18 \AA$ and $R=R_{x}=25 \AA$, umbrella sampling MD runs were carried out in a a number of windows, starting from $\theta=33^{\circ}$ to $\theta=53^{\circ}$, that resulted in well overlapping histograms. For ACI (MOLI) at $R_{0}$ the windows were centered around the following $\theta_{i}$ angels (measured in degrees): 33, 35, 37, 38, 39, 40, 41, 42, 43, 45, 47, 49, 51 and 53 (35, 36, 37, 38, 39, 40, 41, 42, 43, $45,47,49,51$ and 53$)$. For $R_{x}$ the choice of window positions were similar. The target angle was enforced by applying a $2 \mathrm{D}$ harmonic guiding potential $V_{i, j}(\theta, R)=k_{i}\left(\theta-\theta_{i}\right)^{2} / 2+k_{R}\left(R-R_{j}\right)^{2} / 2$ with $j=0, x, k_{R}=80 \mathrm{kcal} / \mathrm{mol} \AA^{2}$, and $k_{i}$ tuned between $8-10 \times 10^{3} \mathrm{kcal} / \mathrm{mol} \mathrm{rad}^{2}$ for achieving optimal sampling in each window. After exhaustive testings, simulations were performed for $0.7 \mathrm{~ns}$ for each window. Only the last $0.5 \mathrm{~ns}$ parts of the trajectories were used to construct the histograms. Test runs confirmed that complete sampling in each window is achieved on this time scale. The $k_{i}, \theta_{i}$ and $\theta$ histograms have been used in the WHAM equations to calculate the PMF $U(\theta \mid R)$ as described in Sec. 3.3.

\section{Results}

\subsection{Equilibrium MD simulations of MOLI and ACI dimers}

In order to test their reliability and usefulness, we have monitored the time evolution of the reaction coordinates $R$ and $\theta$, during 5 ns long equilibrium MD simulations of two LH2 subunit dimers, one from MOLI (Rs. molischianum) and one for ACI (Rps. acidophila). The simulated systems were prepared as described in Sec. 2. The calculated values of the reaction coordinates, i.e., $R-$ the separation, within the plane of the membrane, between the center-of-mass (COM) of the $\alpha \beta$-polypeptide pairs in the two subunits, and $\theta$ - the angle between the projection on the membrane plane of the lines that connect the COM of the $\alpha$ - and $\beta$-polypeptides for each subunit (cf. Fig. 3), depends on the selection of atoms used to determine the COMs. Through extensive testing, we found that the most stable reaction coordinates correspond to the situation in which only the heavy atoms of the trans-membrane helical (TMH) domain of the $\alpha \beta$-apoproteins are considered. In this case, the reaction coordinate assumes well defined mean equilibrium values. The distribution histograms of 
the values of the reaction coordinates for the last $2 \mathrm{~ns}$ of the MD trajectories are shown in Fig. 4. The peak positions in the histograms (corresponding to the most probable values of the reaction coordinates) in Fig. 4 are $R_{0} \approx$ $18 \pm 0.08 \AA, \theta_{0} \approx 42.4^{\circ} \pm 1.7^{\circ}$ for MOLI, and $R_{0} \approx 17.8 \pm 0.15 \AA, \theta_{0} \approx$ $38.1^{\circ} \pm 1.4^{\circ}$ for ACI. For comparison, Fig. 4 also includes the corresponding histograms for a complete Rs. molischianum LH2 ring, obtained from the last 3 ns part of a 5 ns long equilibrium MD run, and with $R_{0} \approx 18.2 \pm 0.2 \AA$ and $\theta_{0}=45 \pm 2.1^{\circ}$. According to these results, we find that for both MOLI and ACI subunit dimers, $\theta_{0}$ is about $2-3^{\circ}$ smaller than the compared theoretically expected value (i.e., $45^{\circ}$ for MOLI and $40^{\circ}$ for ACI). However, there is a significant difference in the most probable angle of about $4^{\circ}$, close to the theoretically expected difference of $5^{\circ}$. For the MOLI LH2 ring $\theta_{0}$ coincides with the expected $45^{\circ}$, albeit the fluctuations in the angle are noticeably larger than for the subunit dimers.

Furthermore, fluctuations in $R$ are much smaller than fluctuations in $\theta$, and the mean value $R_{0}$ is essentially the same (within less than half of one $\AA$ ) for all three systems. If one tries to extend the protein atoms selection in the definition of the reaction coordinates, e.g., by including the $\mathrm{CT}$ (C-terminus) and NT (N-terminus) domains of the $\alpha \beta$-polypeptides, the resulting reaction coordinates become ill defined especially due to the sharp increase in the magnitude of the fluctuations. In such cases, we find that during test MD simulations (not shown) the fluctuations both in $\theta$ and $R$ increase by more than a factor of 2 . These findings are consistent with the knowledge that in general the TMH regions of membrane proteins are more rigid than the outer membrane parts.
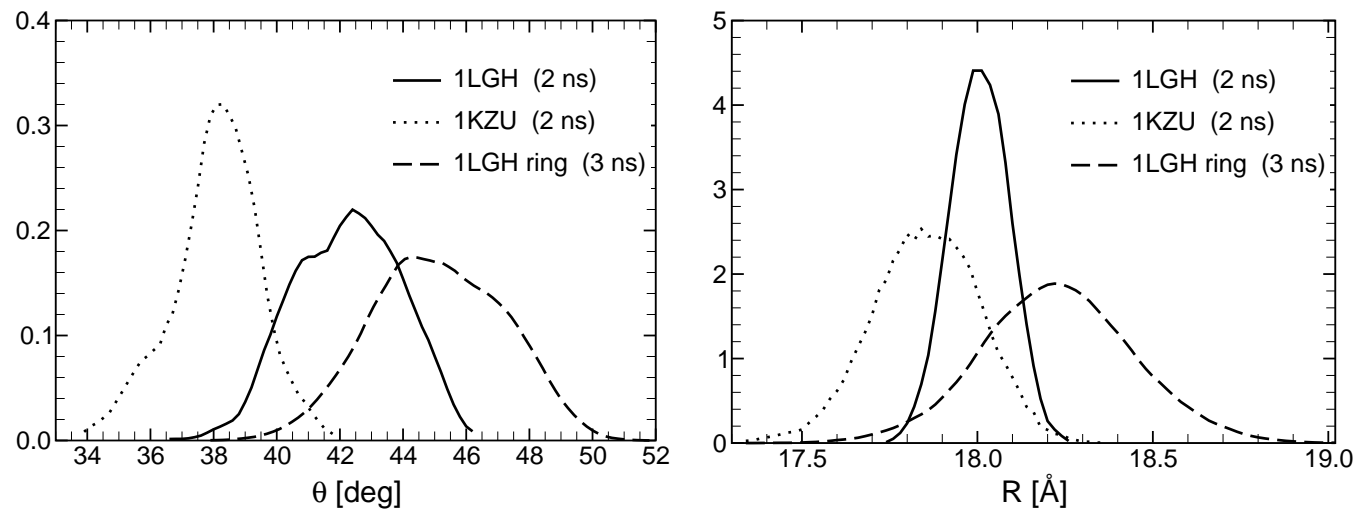

Fig. 4. Histograms of the reaction coordinates $\theta$ (left) and $R$ (right) corresponding to equilibrium MD simulations as follows: MOLI dimer, 2 ns run (solid curve), ACI dimer, 2 ns run (dashed curve), and MOLI LH2 ring, 3 ns (long-dashed curve). 


\subsection{Forced detachment of the subunits using SMD}

Next we employ the reaction coordinates introduced above to investigate the relationship between the spatial separation and relative orientation of the two LH2 subunits as a result of their forced detachment. In principle, such a study requires the knowledge of the $2 \mathrm{D}$ potential of mean force (PMF) $U(R, \theta)$, that describes the statistical mechanical state of the system as a function of the two reaction coordinates. However, a brute force determination of the PMF (by direct application of either methods of calculating PMFs described in Sec. 2) is computationally prohibitively expensive.

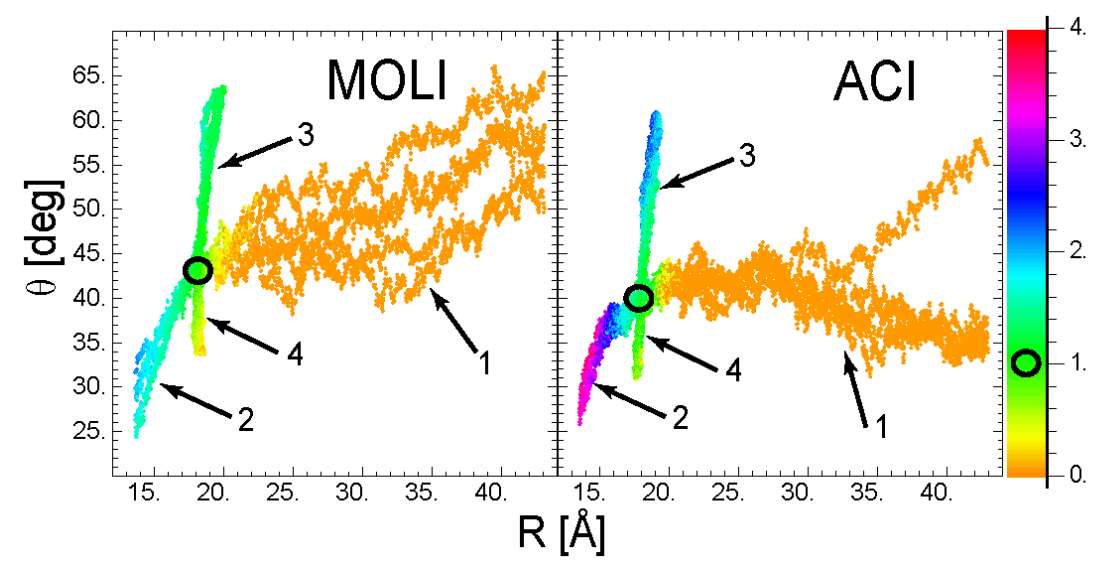

Fig. 5. Two dimensional density plot of the volume overlap of the transmembrane protein regions of MOLI (left) and ACI (right) dimers along the reaction coordinates $\theta$ and $R$ recorded in the SMD simulations described in the text; the trajectories corresponding to the four different sets of simulations are indicated by numbered arrows. The volumes are relative to the value corresponding to the equilibrium reaction coordinates $\theta_{0}$ and $R_{0}$ (see text), marked by the small circle.

Thus, to gain some insight into the mechanism that governs the relationship between the relative distance and orientation of the LH2 subunit dimer during the forced separation or compression of the subunits, we have conducted four sets of 5 SMD simulations each, starting from initial states that coincide with the last frames of the equilibrium simulations shown in Fig. 4, and characterized by $R_{0}=18.2 \AA$ and $\theta_{0}=43.7^{\circ}$ for MOLI and $R_{0}=18.1 \AA$ and $\theta_{0}=41^{\circ}$ for ACI. These starting values are marked by small circles in Fig. 5. In the first (second) set of simulations $\theta$ was unconstrained while $R$ was increased (decreased) with a constant rate of $v_{R}=0.1 \AA / \mathrm{ps}$, by applying a harmonic guiding potential $V(\tilde{R} \mid R)=k_{R}(\tilde{R}-R)^{2} / 2$, as described in Sec. 2, with $k_{R}=500 \mathrm{kcal} / \mathrm{mol} \AA^{2}$. Similarly, in the third (fourth) set of simulations $R$ was unconstrained while $\theta$ was increased (decreased) with $v_{\theta}=0.1 \mathrm{deg} / \mathrm{ps}$ through a harmonic guiding potential $V(\tilde{\theta} \mid \theta)=k_{\theta}(\tilde{\theta}-\theta)^{2} / 2$ with $k_{\theta}=5 \times 10^{4} \mathrm{kcal} / \mathrm{mol} \mathrm{\textrm {rad } ^ { 2 }}$. In these potentials, $R$ and $\theta$ are the target values of the reaction coordinates while $\tilde{R}$ and $\tilde{\theta}$ represent the instantaneous 
value of the reaction coordinate as determined from the corresponding atomic coordinates. The SMD trajectories in the reaction coordinate plane (i.e., the loci of points with coordinates $\{R(t), \theta(t)\})$ are shown in Fig. 5 for both MOLI and ACI. The different trajectories belonging to different sets of simulations are indicated by numbered arrows. Trajectory points are color-coded according to the current volume overlap of the TM parts of the two subunits, normalized to the corresponding initial state value.

The reaction coordinate trajectories exhibit distinctive features for each set of simulations, with manifest differences between the two systems.

As soon as the separation $R$ between the two subunits is increased (set 1) past $R_{0}$, the TMH domains of the $\alpha \beta$-apoproteins separate for both MOLI and ACI, an event which is accompanied by a noticeable increase in the fluctuations of the angle reaction coordinate. While in the case of ACI, the separation of the N-terminal and TMH domains seem to occur simultaneously as $R$ increases, in contrast, for MOLI, the N-terminal domains of the subunits do not detach until $R$ becomes larger than $35 \AA$. On the other hand, the C-terminal domains remain connected in both systems even for separations as large as $40 \AA$. This difference may also be responsible for the profile of the trajectories in set 1 . For MOLI, as $R$ increases, the trajectories cluster in three well distinguished paths, with occasional partial overlaps and are subject to large $\theta$ fluctuations. This suggests that the PMF $U(\theta \mid R)$, for a given $R>R_{0}$, has a broad global minimum with several (at least three) local minimums separated by relatively small potential barriers. The location of the minimum is shifted to $\theta>\theta_{0}$ values (see below). On the other hand, for ACI, as $R$ increases, the trajectories remain clustered (albeit with enhanced $\theta$ fluctuations) suggesting that the PMF $U(\theta \mid R)$ has a potential well that is broader than the one for $R_{0}$. However, the dramatic deviation of one of the trajectories from the rest for $R \gtrsim 35 \AA$ suggests that for larger separation $U(\theta \mid R)$ may develop a structure with at least two well separated local minimums, with one equilibrium angle smaller and the other one larger than $\theta_{0}$.

The behavior of the reaction coordinate trajectories for the simulations in sets 2, 3 and 4 are qualitatively similar for both MOLI and ACI. Already a few $\AA$ compression of $R$ (set 2) increases the overlap of the protein subunits several times, accompanied by a decrease of the angle variable. The trajectories nicely overlap, indicating that the corresponding PMF $U(\theta \mid R)$ is similar to the one corresponding to $R_{0}$, with the minimum shifted towards a smaller angle than $\theta_{0}$.

Finally, it is remarkable that for the simulations in sets 3 and 4 , in which $R$ is unconstrained, the latter shows only a slight increase with respect to $R_{0}$ as the angle is increased (decreased) by as much as $20^{\circ}\left(10^{\circ}\right)$. Thus, based on the results of our SMD simulations, one may conclude that in general forced 


\begin{tabular}{|l|l|l|l|}
\hline $\begin{array}{l}\text { subunit 1 } \\
\text { residue }\end{array}$ & $\begin{array}{l}\text { subunit 2 } \\
\text { residue(s) }\end{array}$ & $\begin{array}{l}\text { average distance } \\
\text { for breaking links }\end{array}$ & contact region \\
\hline \hline$\alpha_{1} \mathrm{GLY}_{+8}$ & $\alpha_{2} \mathrm{ALA}_{+16}$ & $>40 \AA$ & C-terminus \\
$\beta_{1} \mathrm{LYS}_{+7}$ & $\alpha_{2} \mathrm{ALA}_{+16}, \mathrm{SER}_{+19}, \mathrm{ALA}_{+20}$ & $>40 \AA$ & C-terminus \\
$\beta_{1} \mathrm{PRO}_{+8}$ & $\alpha_{2} \mathrm{GLY}_{+15}, \mathrm{SER}_{+19}$ & $>40 \AA$ & C-terminus \\
$\beta_{1} \mathrm{TRP}_{+9}$ & $\alpha_{2} \mathrm{GLY}_{+15}$ & $>40 \AA$ & C-terminus \\
$\alpha_{1} \mathrm{PHE}_{+9}$ & $\alpha_{2} \mathrm{ILE}_{+12}$ & $36-38 \AA$ & C-terminus \\
$\beta_{1} \mathrm{ARG}_{-32}$ & $\beta_{2} \mathrm{LEU}_{-27}$ & $32-35 \AA$ & N-terminus \\
$\beta_{1} \mathrm{LEU}_{-30}$ & $\alpha_{2} \mathrm{SER}_{-18}$ & $21-22 \AA$ & N-terminus \\
$\alpha_{1} \mathrm{VAL}_{-22}$ & $\alpha_{2} \mathrm{PRO}_{-14}, \mathrm{SER}_{-18}$ & $21-24 \AA$ & TM \\
\hline
\end{tabular}

Table 1

Average separation distances at which the most important inter-residue links between two LH2 subunits of Rs. molischianum are broken in SMD simulations.

Table 2

\begin{tabular}{|l|l|l|l|}
\hline $\begin{array}{l}\text { subunit } 1 \\
\text { residue }\end{array}$ & $\begin{array}{l}\text { subunit 2 } \\
\text { residue(s) }\end{array}$ & $\begin{array}{l}\text { average distance } \\
\text { for breaking links }\end{array}$ & contact region \\
\hline \hline$\alpha_{1} \mathrm{THR}_{+7}$ & $\alpha_{2} \mathrm{TRP}_{+14}, \mathrm{GLN}_{+15}$ & $>40 \AA$ & C-terminus \\
$\alpha_{1} \mathrm{THR}_{+8}$ & $\alpha_{2} \mathrm{GLN}_{+15}$ & $>40 \AA$ & C-terminus \\
$\alpha_{1} \mathrm{TRP}_{+9}$ & $\alpha_{2} \mathrm{TRP}_{+14}, \mathrm{GLN}_{+15}$ & $>40 \AA$ & C-terminus \\
$\beta_{1} \mathrm{PRO}_{+8}$ & $\alpha_{2} \mathrm{GLN}_{+15}$ & $>40 \AA$ & C-terminus \\
$\beta_{1} \mathrm{TRP}_{+9}$ & $\alpha_{2} \mathrm{TYR}_{+13}$ & $34-38 \AA$ & C-terminus \\
$\alpha_{1} \mathrm{VAL}_{-21}$ & $\alpha_{2} \mathrm{ALA}_{-18}, \mathrm{PRO}_{-13}$ & $20-22 \AA$ & N-terminus \\
$\alpha_{1} \mathrm{VAL}_{-22}$ & $\alpha_{2} \mathrm{ALA}_{-18}, \mathrm{PRO}_{-19}$ & $21-27 \AA$ & N-terminus \\
$\alpha_{1} \mathrm{ALA}_{+2}$ & $\alpha_{2} \mathrm{LEU}_{+4}$ & $23-25 \AA$ & TM \\
\hline
\end{tabular}

Average separation distances at which the most important inter-residue links between two LH2 subunits of Rps. acidophila are broken in SMD simulations.

rotation of the relative orientation of the subunits has only very limited effect on their spatial separation. On the other hand, the modification of the distance between the subunits in general has strong impact on the angle between the subunits. This conclusion also provides support to the notion that the preferred angle between LH2 subunits is mainly determined by contact interactions between subunits.

Steered molecular dynamics simulations also provide insights into the key interactions between the subunits: as two subunits are pulled apart, the links between the subunits break from the weakest to the strongest. We consider a 
link broken, if the distance between the closest contact between two residues becomes larger than $3 \AA$. A list of strongest links, as presented in Tables 1 and 2, provides qualitative information on the basis of SMD simulation data. With increased sampling, more refined analysis in terms of e.g. interaction energies or free energy barriers would become possible. Inspection of the Tables shows that all links in the TMH domain become separated for distances larger than $R=25 \AA$ for both cases. In the N-terminal domain, all links become separated for distances around $25 \AA$ for ACI and around $35 \AA$ for MOLI. This is consistent with the overlapping volume data shown in Fig. 5. On the other hand, there is one group of links in the C-terminal domain for both ACI and MOLI that persists even for separations larger than $40 \AA$. This group represents the strongest interactions conferring overall stability of the binding of the two subunits. An immediately recognizable difference is that most of the links in ACI are between the $\alpha$-apoproteins while they are between between the $\beta 1$ and $\alpha 2$ in MOLI.

\subsection{Calculation of PMF $U(\theta, R)$}

Our focus in the present manuscript is to investigate the interaction angle between two subunits. To this end, we have determined two 1D PMFs $U_{i}\left(\theta \mid R_{i}\right)$ along $\theta$ by using umbrella sampling and WHAM, as described in Sec. 2.

The calculations were performed for both MOLI and ACI, for two representative separations, i.e, the equilibrium $R_{0}=18 \AA$ and $R_{x}=25 \AA$. While the choice for the equilibrium value is obvious, the reason for the $R_{x}$ selection is that at this particular distance MOLI and ACI are in qualitatively different separation states. While, for $R_{x}$, the TMH domains of the $\alpha \beta$-polypeptides are already separated in both MOLI and ACI, the N-terminal domain is fully separated only in ACI, but not in MOLI; the C-terminal domains are still in contact for both systems.

The computed PMFs, using umbrella sampling and WHAM, are shown in Fig. 6. For $R_{0}$, as expected, the PMF for both MOLI and ACI exhibits a nearly parabolic lineshape with a minimum that coincides with the position of the peak $\theta_{0}$ in the corresponding equilibrium angle distribution histogram in Fig. 4a $\theta_{p}$. In fact, the PMFs calculated from those histograms as $U_{0}(\theta) \propto$ $-k_{B} T \ln \left[p_{0}(\theta)\right]$ matches rather well the bottom of the full PMF obtained from umbrella sampling and WHAM (data not shown). At the equilibrium distance, the PMF of ACI is narrower than that of MOLI, corresponding to stronger angular constraints for ACI compared to MOLI. This is consistent with the finding from the SMD simulations that the fluctuations in the angular reaction coordinate are smaller for ACI than for MOLI. Compared to the $R_{0}$ case, for $R_{x}$ the PMF for both systems widens up and acquires additional features. 


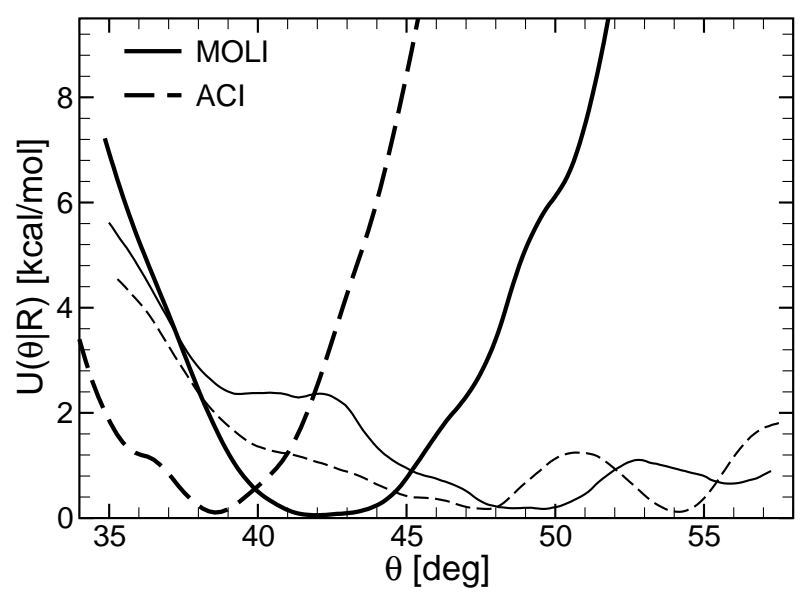

Fig. 6. Calculated potentials of mean force $U(\theta \mid R)$ for both MOLI (solid lines) and ACI (dashed lines) dimers. The thick (thin) curves correspond to $R=18 \AA$ $(R=25 \AA)$.

For MOLI, the PMF exhibits a small plateau at angles around $40^{\circ}$, and a steep downhill region for $\theta \gtrsim 42^{\circ}$ that ends in a broad minimum around $49^{\circ}$, followed by a modest potential barrier at $\sim 53^{\circ}$. These features are consistent with the SMD results. Indeed, the angle spread of the SMD trajectories at $R_{x}$ extends from $\sim 40^{\circ}$ to $\sim 55^{\circ}$. The steep potential barrier in the PMF at $\lesssim 40^{\circ}$ explains the lack of trajectory points below this value. Also, the higher trajectory points density along the plateau region and the broad minimum is expected. For ACI, the PMF for $R_{x}$ shows a $\sim 10^{\circ}$ wide, rather flat region starting from $39^{\circ}$. The fact that the corresponding SMD trajectory points are clustered only in the interval $38^{\circ}<\theta<44^{\circ}$ does not conflict with the PMF data but raises the question why there are no trajectory points up to angles of $\sim 50^{\circ}$ ? There are several possible answers. First, the small number of SMD trajectories may not provide a proper sampling of the angles for $R_{x}$. Secondly, the $\theta$ self diffusion coefficient may be very small, so that a flat PMF does not lead to significant dispersion. The second well in the PMF at $54^{\circ}$ suggests that eventually a second branch of trajectories may appear oriented towards higher angle values as it appears indeed for $R>32 \AA$.

\section{Discussion and Conclusions}

Understanding the assembly of a protein complex requires that one addresses a set of interrelated questions: (1) What is the temporal order in which parts are put together? (2) What interactions stabilize the protein complex or parts of the complex? (3) What factors govern the (reproducible) stable geometry of the complex?

In this manuscript, we focus on the assembly of a two-subunit complex from 
two fully formed $\alpha \beta$-subunits with three BChl and one carotenoid bound. Spectroscopic observations support the assumption that this is an important step in the formation of light harvesting complexes as they show a progression from monomeric BChl $(777 \mathrm{~nm})$ to a BChl dimer bound to an $\alpha \beta$-subunit (820 $\mathrm{nm})$ to a complex formed of two $\alpha \beta$-subunits $(851 \mathrm{~nm})[50,51]$. It is, however, unknown whether carotenoids are already incorporated at this step and how closely the structure of the $\alpha \beta$-subunit at this point matches the structure of the $\alpha \beta$-subunit in the crystal structures, from which we took the coordinates.

An initial analysis of fluctuations in a two-subunit complex equilibrated in a lipid-water environment revealed that the hydrophobic core region is rather rigid. Fluctuations in the centers-of-mass of the core regions in free molecular dynamics runs are small enough to allow for a meaningful definition of global coordinates (distance, angle) of the subunits as introduced above.

In order to probe the assembly process, we performed two sets of calculations with different philosophies. In one set of calculations, we constrain the subunits at different angles and allow the system to equilibrate under this constraint. From such umbrella sampling, we then extract information about the free energy profile as a function of angle. Pulling two subunits apart through steered molecular dynamics gives complementary information into key events of the binding/unbinding process and a rough order of interaction strengths.

\subsection{Preferred angle}

Our calculations show that two subunits in van-der-Waals contact (center-tocenter distance of $18 \AA$ ) arrange at a preferred angle with each other. Figs. 7a,c show the transmembrane domains of subunits in the preferred arrangements at this distance. For subunits of LH2 from Rs. molischianum, the minimum of the free energy profile (PMF; cf. Fig. 6) is located at about $42.5^{\circ}$, whereas for Rps. acidophila subunits, the minimum is located at $38.5^{\circ}$. The free energy profiles closely match parabolic profiles. Whereas changes of about one degree around the minimum position carry only a small energetic penalty, it requires about $3 \mathrm{kcal} / \mathrm{mol}$ to force two subunits from Rps. acidophila into the angle of $42.5^{\circ}$ preferred by subunits from Rs. molischianum. Likewise, about $2 \mathrm{kcal} / \mathrm{mol}$ are required to force two subunits from Rs. molischianum into the angle of $38.5^{\circ}$ preferred by subunits from Rps. acidophila. This suggests that the preferred angle between the subunits plays an important role in guiding the assembly of light harvesting complexes into a particular ring size or oligomerization state. However, the preferred angles, while comparing well to the theoretically expected angles of $45^{\circ}$ (8-fold symmetry) and $40^{\circ}$ (9-fold symmetry) are somewhat smaller than these. It is possible that the theoretically expected preferred angle is only assumed once each subunit is in contact 
with a subunit on either side, as would be the case in a ring geometry. The fact that the average interaction angle in a free molecular dynamics simulation run of a completed ring is $44.8^{\circ}$, much closer to the expected $45^{\circ}$, supports this assumption.

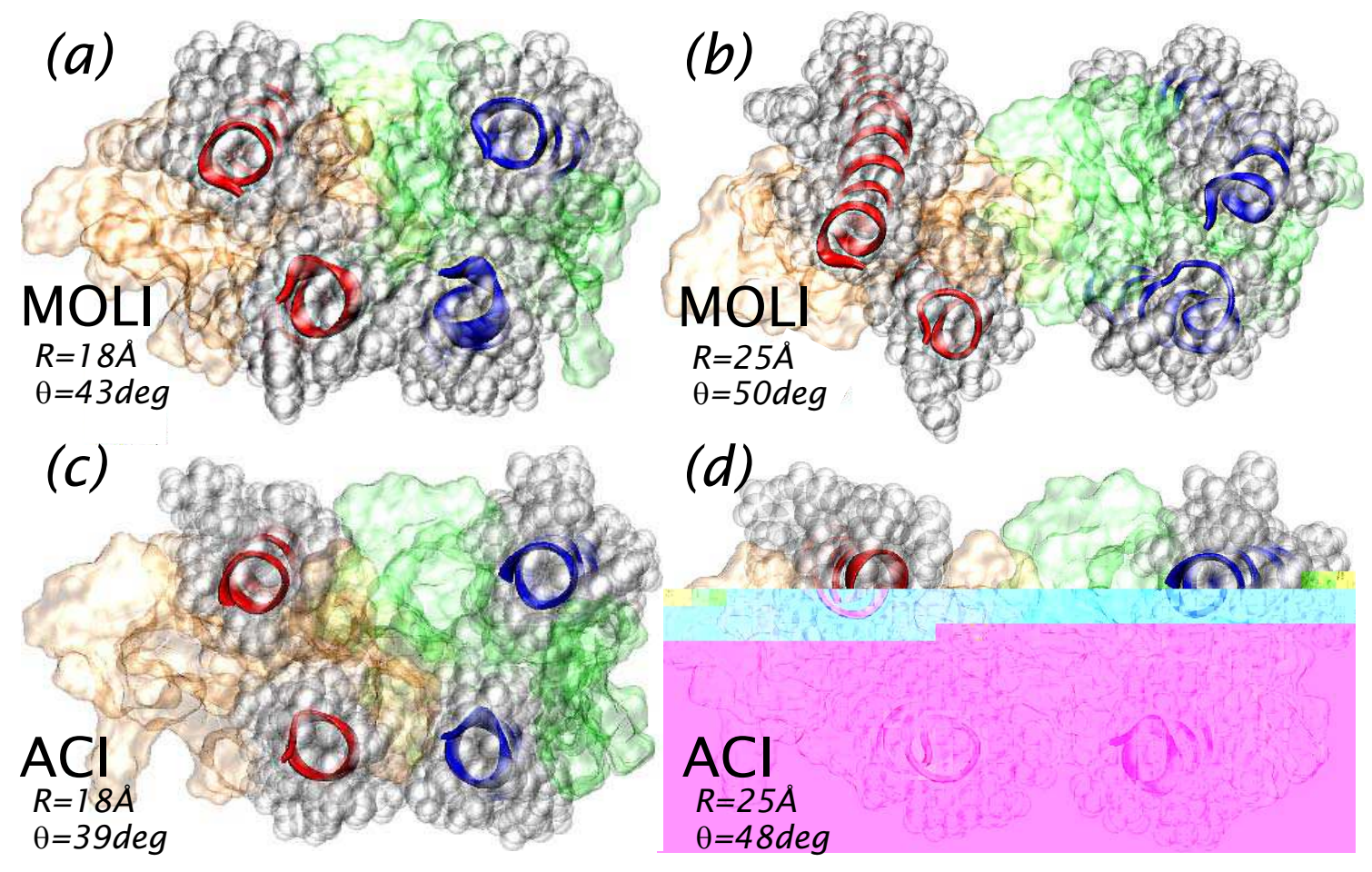

Fig. 7. Top view from the N-terminus of the transmembrane region of MOLI (a and b) and ACI (c and d) dimers. The values of the corresponding reaction coordinate $R$ and $\theta$ are indicated for each case. The backbone of the transmembrane helices are shown in cartoon representation. The sidegroups of the proteins and the enclosed cofactors (BChls and Car) are shown in transparent space-filling representation.

To see whether the surface contact between the two subunits is important in defining a preferred angle, we performed additional calculations where two subunits are constrained to a center-to-center distance of $25 \AA$. At this distance, subunits of Rs. molischianum are still connected at both their C- and $\mathrm{N}$-terminal domains, and subunits of Rps. acidophila are connected at their C-terminus only. However, the $\alpha \beta$ polypeptides in the transmembrane region are no longer in contact for either subunit pairs (cf. Fig. 7). In this case, the free energy decreases at larger angles with considerably more shallow minima than the free energy profiles at the equilibrium distance of $18 \AA$.

These findings suggest that the exact preferred angle between two subunits is largely defined by the surface interactions in the transmembrane region. Once the $\alpha \beta$-polypeptides in the transmembrane region become disconnected, the angular variation increases significantly (see Fig. 5). However, angular constraints, albeit more relaxed, still exist even at larger separations of the subunits. 
Interestingly, the angle between two subunits is more constrained in Rps. acidophila than in Rs. molischianum. The parabola of the free energy profile at equilibrium distance $(18 \AA)$ is narrower for Rps. acidophila than for Rs. molischianum. Moreover, the five trajectories of Rps. acidophila during the steered molecular dynamics simulations show relatively little angular spread after the transmembrane regions are disconnected and even after the links in the $\mathrm{N}$ terminal domain are broken. Much more angular spread can be observed in the case of equivalent steered molecular dynamics runs for Rs. molischianum, even for cases when the links in both the $\mathrm{C}$ - and N-terminal regions are still intact. As stated above, the fast pulling and small sample size during this simulation raises a caveat, as the configuration space may not have been sampled well.

If a more complete sampling of the $2 \mathrm{D}$ free energy surface confirms the observed differences in angular constraints, it would suggest that in Rps. acidophila a motif in the C-terminal domain plays a role in constraining the angle between subunits in addition to the hydrophobic surface contacts. One can speculate that this double constraint of the angle could be the reason why 9-rings are much more prevalent among LH2 complexes than rings of other sizes.

\subsection{Stabilizing links}

In addition to exploring the factors constraining the angle between two subunits, the main focus of this publication, we chose computationally inexpensive SMD calculations to obtain qualitative, but not quantitative information about the factors stabilizing the connection between two subunits.

The results from these calculations suggest that links in the terminal domains play an important role in stabilizing the complex. These links break last as subunits are pulled apart and thus represent the strongest interactions between the two subunits. The strongest interactions are all found in the C-terminal domain. Interactions in the TMH domain are weakest (cf. Tables 1,2). Only a limited number of experiments address the question of the requirements for formation of a light harvesting complex; most mutagenesis experiments are concerned with the formation of a $\alpha \beta$-subunit. In experimental studies that led to successful formation of a complete light harvesting complex, the terminal domains were taken from native sequences, thus offering little insight into what parts of the terminal domains are required for successful formation of complete light-harvesting complexes $[24,25,30]$. Recently, Braun et al. demonstrated formation of functional light harvesting complexes from polypeptides in which all amino acids in the TMH domain except for the ligating $\mathrm{His}_{0}$ were replaced by alternating pairs of alanin and leucine residues [31]. An addition 
of four amino acids in the C-terminal domain resulted in a complete loss of light harvesting complex formation. This study supports a crucial role the C-terminal domain in connecting subunits to form complete light harvesting complexes, whilst indicating a less important role of the TMH domain.

So far, we have discussed the two dimensions distance and angle separately from each other. Interestingly, the differences between Rs. molischianum and Rps. acidophila suggest different rules by which distances and angles are stabilized. In Rs. molischianum, separation of two subunits appears to require more energy as the N-terminal domains stay connected far beyond distances where they are disconnected in Rps. acidophila. On the other hand, as discussed above, the angle is more constrained in Rps. acidophila. A proper discussion of the interplay between constraints in angle and distance, requires evaluation of the full two-dimensional PMF, which is beyond the scope of this publication.

\subsection{Outlook}

Our results suggest a guided key-lock principle in the assembly of light harvesting complexes. Interactions in the terminal domains, in particular the C-terminal domain serve as 'hooks' that connect the subunits; however, they do not constrain the angle between the subunits very strongly, although in the case of Rps. acidophila, the C-terminus may assist in constraining the angle. Once the surfaces of the subunits, in particular the BChl co-factors, start to interlock in the hydrophobic core region, the angle between the subunits becomes well defined.

To our knowledge, no previous theoretical study has attempted to address the question as to why some LH2 complexes form 8-rings, while most form 9-rings. It is therefore a very intriguing result that we found a distinct difference in the preferred angle between two subunits that closely matches the expected angle difference of $5^{\circ}$ between an 8-ring in $R$ s. molischianum and a 9-ring in Rps. acidophila.

This result is a promising starting point towards understanding the rules by which light harvesting complexes assemble into rings of defined sizes. Obviously, many questions remain to be answered, for example: Do the rules underlying assembly of two subunits remain the same when many subunits assemble? In other words, are there effects of different subunit concentrations, leading to phase transitions between different ring sizes (see e.g., [52])? What is the role of transcription factors and chaperones for assembly in vivo?

Genetic manipulation is a powerful tool to obtain information about the assembly of light harvesting complexes. Several experimental studies attempting to modify the size of an LH ring by truncating or swapping C-terminal do- 
mains $[15,30]$ did not observe any alterations of the ring size, although changes in the hydrogen bonding could be observed. Olsen et al. speculate that steric constraints involving BChls, especially B800 BChls play a role in determining the ring size [30]. Our study supports this view as it suggests an important role of surface contacts in the hydrophobic region in constraining the angle between two subunits. Most contacts in the transmembrane region are made by the BChls ligated to the $\alpha$ and $\beta$ polypeptides. Thus, constraining the angle between two subunits requires positioning BChls into an orientation that in turn favors a particular angle between two subunits due to steric constraints. Amino acid substitutions will therefore only have an indirect effect on the ring size, by repositioning the (conserved) BChls into different orientations through changes in the binding pocket or ligation pattern. It is therefore by no means straightforward to predict the effects of amino acid substitutions on the ring size.

Through homology modeling of light-harvesting complexes, one can, in principle, emulate the genetic manipulation process. Based on such in silico mutants and using the calculation techniques presented here, one can then calculate the preferred interaction angles of complexes with amino acid deletions or substitutions. The goal of such calculations would be to predict new complexes for which, e.g. a 10-ring or 7-ring architecture is expected. Using established in vivo assembly experiments and AFM imaging techniques, one can test whether these de novo designs indeed form the expected ring sizes.

A successful demonstration of the predictive power of molecular dynamics combined with non-equilibrium techniques could pave the way towards understanding the principles underlying the assembly of multimeric membrane protein complexes. Such understanding would have relevance in controlling nanodevices built from photosynthetic materials. Furthermore, formation of multimeric protein complexes occurs in many membrane protein complexes. Combining in silico with experimental genetic manipulation could therefore yield information on the assembly not only of light harvesting complexes, but of membrane protein complexes in general.

\section{Acknowledgments}

This work was supported by grants from the University of Missouri Research Board (LJ and IK), the Institute for Theoretical Sciences, a joint institute of Notre Dame University and Argonne National Laboratory, and the U.S. Department of Energy, Office of Science through contract No. W-31-109-ENG38 (IK and TR). 


\section{References}

[1] X. Hu, T. Ritz, A. Damjanovic, F. Autenrieth, K. Schulten, Photosynthetic apparatus of purple bacteria., Q Rev Biophys 35 (1) (2002) 1-62.

[2] R. Cogdell, A. Gardiner, A. Roszak, C. J. Law, J. Southall, N. W. Isaacs, Rings, ellipses and horseshoes: How purple bacteria harvest solar energy, Photosynthesis Research 81 (2004) 207-214.

[3] S. Scheuring, J. Sturgis, V. Prima, A. Bernadac, D. Lévy, J. Rigaud, Watching the photosynthetic apparatus in native membranes., Proc. Natl. Acad. Sci. USA 101 (2004) 11293-11297.

[4] S. Bahatyrova, R. Frese, C. Siebert, J. Olsen, K. van der Werf, R. van Grondelle, R. Niederman, P. Bullough, C. Otto, C. Hunter, The native architecture of a photosynthetic membrane., Nature 430 (2004) 1058-1062.

[5] G. Fleming, R. van Grondelle, Femtosecond spectroscopy of photosynthetic light-harvesting systems, Curr. Opin. Struct. Biol. 7 (1997) 738-748.

[6] T. Sundstrom, V.and Pullerits, R. van Grondelle, Photosynthetic lightharvesting: reconciling dynamics and structure of purple bacterial LH2 reveals function of photosynthetic unit, J. Phys. Chem. B 103 (1999) 2327-2346.

[7] T. Ritz, S. Park, S. K., Kinetics of excitation migration and trapping in the photosynthetic unit of purple bacteria., Journal of Physical Chemistry B 105 (2001) 8259-8267.

[8] T. Ritz, A. Damjanovic, K. Schulten, The quantum physics of photosynthesis., Chemphyschem 3 (3) (2002) 243-8.

[9] G. McDermott, S. Prince, A. Freer, A. Hawthornthwaite-Lawless, M. Papiz, R. Cogdell, N. Isaacs, Crystal structure of an integral membrane lightharvesting complex from photosynthetic bacteria., Nature 374 (1995) 517-521.

[10] J. Koepke, X. Hu, C. Muenke, K. Schulten, H. Michel, The crystal structure of the light-harvesting complex II (B800-850) from Rhodospirillum molischianum., Structure 4 (1996) 581-597.

[11] M. Papiz, S. Prince, T. Howard, R. Cogdell, N. Isaacs, The structure and thermal motion of the B800-850 lh2 complex from rps. acidophila at 2.0 Åresolution and $100 \mathrm{k}$ : new structural features and functionally relevant motions., J. Mol. Biol. 326 (2003) 1523-1538.

[12] G. Montoya, M. Cyrklaff, I. Sinning, Two-dimensionalcrystallization and preliminary structure analysis of light harvesting II (B800-850) complex from the purple bacterium Rhodovulum sulfidophilum., J. Mol. Biol. 250 (1995) 1-10.

[13] T. Walz, S. Jamieson, C. Bowers, B. P.A., C. Hunter, Projection structures of three photosynthetic complexes from rhodobacter sphaeroides: LH2 at $6 \stackrel{\circ}{A}$, LH1

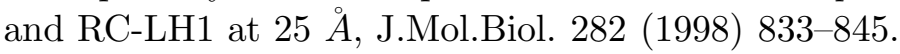


[14] S. Scheuring, J. Seguin, S. Marco, D. Levy, B. Robert, J. Rigaud, Nanodissection and high-resolution imaging of the Rhodopseudomonas viridis photosynthetic core complex in native membranes by AFM., Proc. Natl. Acad. Sci. USA. 100 (2003) 1690-1693.

[15] J. Ranck, T. Ruiz, G. Pehau-Arnaudet, B. Arnoux, F. Reiss-Husson, Two-dimensional structure of the native light-harvesting complex lh2 from Rubrivivax gelatinosus and of a truncated form., Biochim. Biophys. Acta. 1506 (2001) 67-78.

[16] S. Scheuring, F. Reiss-Husson, A. Engel, J.-L. Rigaud, J.-L. Ranck, High resolution topographs of the rubrivivax gelatinosus light-harvesting complex 2., EMBO J. 20 (2001) 3029-3035.

[17] N. Hartigan, H. Tharia, F. Sweeney, A. Lawless, M. Papiz, The 7.5-Åelectron density and spectroscopic properties of a novel low-light b800 lh2 from rhodopseudomonas palustris., Biophys J. 82 (2002) 963-977.

[18] S. Scheuring, J. Rigaud, J. Sturgis, Variable LH2 stoichiometry and core clustering in native membranes of Rhodospirillum photometricum., EMBO J. 23 (2004) 4127-4133.

[19] S. Jamieson, P. Wang, P. Qian, J. Kirkland, M. Conroy, C. Hunter, P. Bullough, Projection structure of the photosynthetic reaction centre-antenna complex of

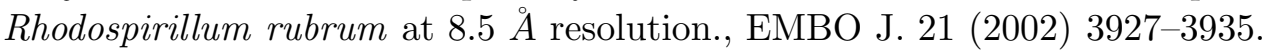

[20] S. Scheuring, J. Seguin, S. Marco, D. Lévy, C. Breyton, B. Robert, J. Rigaud, AFM characterization of tilt and intrinsic flexibility of Rhodobacter sphaeroides light harvesting complex 2 (LH2)., J. Mol. Biol. 325 (2003) 569-580.

[21] A. Roszak, T. Howard, J. Southall, A. Gardiner, C. Law, N. Isaacs, R. Cogdell, Crystal structure of the RC-LH1 core complex from Rhodopseudomonas palustris., Science 302 (2003) 1969-1672.

[22] C. Jungas, J.-L. Ranck, J.-L. Rigaud, P. Joliot, A. Vermeglio, Supramolecular organization of the photosynthetic apparatus of rhodobacter sphaeroides, EMBO J. 18 (1999) 534-542.

[23] P. Parkes-Loach, J. Sprinkle, P. Loach, Reconstitution of the B873 lightharvesting complex of rhodospirillum rubrum from the separately isolated $\alpha$ and $\beta$ polypeptides and bacterichlorophyll $a$, Biochemistry 27 (1988) 27182727 .

[24] J. Todd, P. Parkes-Loach, J. Leykam, P. Loach, In vitro reconstitution of the core and peripheral Light-Harvesting complexes of Rhodospirillum molischianum from separately isolated components., Biochemistry 37 (1998) 17458-17468.

[25] J. Todd, P. Recchia, P. Parkes-Loach, J. Olsen, G. Fowler, P. McGlynn, C. Hunter, P. Loach, Minimal requirements for in vitro reconstitution of the structural subunit of light-harvesting complexes of photosythesis bacteria, Photosynth. Res. 62 (1999) 85-98. 
[26] J. Kehoe, K. Meadows, P. Parkes-Loach, P. Loach, Reconstitution of Core Light-Harvesting Complexes of Photosynthetic Bacteria Using Chemically Synthesized Polypeptides. 2. Determination of Structural Features That Stabilize Complex Formation and Their Implications for the Structure of the Subunit Complex., Biochemistry 37 (1998) 3418-3428.

[27] K. Meadows, P. Parkes-Loach, J. Kehoe, P. Loach, Reconstitution of core lightharvesting complexes of photosynthetic bacteria using chemically synthesized polypeptides. 1. minimal requirements for subunit formation., Biochemistry 37 (1998) 3411-3417.

[28] C. Davis, P. Bustamante, J. Todd, P. Parkes-Loach, P. McGlynn, J. Olsen, L. McMaster, P. Hunter, C.N.and Loach, Evaluation of structure-function relationships in the core light- harvesting complex of photosynthetic bacteria by reconstitution with mutant polypeptides, Biochemistry 12 (1997) 3671-3679.

[29] P. Parkes-Loach, A. Majeed, C. Law, P. Loach, Interactions stabilizing the structure of the core light-harvesting complex (LH1) of photosynthetic bacteria and its subunit (B820)., Biochemistry 43 (2004) 7003-7016.

[30] J. Olsen, B. Robert, C. Siebert, P. Bullough, C. Hunter, Role of the c-terminal extrinsic region of the alpha polypeptide of the light-harvesting 2 complex of rhodobacter sphaeroides: a domain swap study, Biochemistry 42 (2003) 1511415123.

[31] P. Braun, J. Olsen, B. Strohmann, C. Hunter, H. Scheer, Assembly of LightHarvesting bacteriochlorophyll in a model transmembrane helix in its natural environment., J. Mol. Biol. 318 (2002) 1085-1095.

[32] J. Koepke, X. C. Hu, C. Muenke, K. Schulten, H. Michel, The crystal structure of the light-harvesting complex ii (b800-850) from rhodospirillum molischianum, Structure 4 (5) (1996) 581-597.

[33] S. M. Prince, M. Z. Papiz, A. A. Freer, G. McDermott, A. M. HawthornthwaiteLawless, R. J. Cogdell, N. W. Isaacs, Apoprotein structure in the lh2 complex from rhodopseudomonas acidophila strain 10050: modular assembly and protein pigment interactions, J. Mol. Biol. 268 (2) (1997) 412-423.

[34] D. Frenkel, B. Smit, Understanding Molecular Simulation From Algorithms to Applications, Academic Press, California, 2002.

[35] H. Risken, The Fokker-Planck Equation: Methods of Solution and Applications, 3rd Edition, Springer-Verlag Telos, 1996.

[36] B. Roux, The calculation of the potential of mean force using computersimulations, Comput. Phys. Commun. 91 (1-3) (1995) 275-282.

[37] D. Frenkel, B. Smit, Extension to the weighted histogram analysis method: combining umbrella sampling with free energy calculations, Biophys. J. 81 (3) (2001) 1295-1313. 
[38] S. Kumar, D. Bouzida, R. H. Swendsen, P. A. Kollman, J. M. Rosenberg, The weighted histogram analysis method for free-energy calculations on biomolecules .1. the method, J. Comput. Chem. 13 (8) (1992) 1011-1021.

[39] B. Isralewitz, J. Baudry, J. Gullingsrud, D. Kosztin, K. Schulten, Steered molecular dynamics investigations of protein function, J. Mol. Graphics Modell. 19 (1) (2001) 13-25.

[40] H. Grubmuller, B. Heymann, P. Tavan, Ligand binding: Molecular mechanics calculation of the streptavidin biotin rupture force, Science 271 (5251) (1996) 997-999.

[41] C. Jarzynski, Nonequilibrium equality for free energy differences, Phys. Rev. Lett. 78 (14) (1997) 2690-2693.

[42] S. Park, K. Schulten, Calculating potentials of mean force from steered molecular dynamics simulations, J. Chem. Phys. 120 (13) (2004) 5946-5961.

[43] G. Hummer, A. Szabo, Free energy reconstruction from nonequilibrium singlemolecule pulling experiments, Proc. Natl. Acad. Sci. U. S. A. 98 (7) (2001) $3658-3661$.

[44] J. Gore, F. Ritort, C. Bustamante, Bias and error in estimates of equilibrium free-energy differences from nonequilibrium measurements, Proc. Natl. Acad. Sci. U. S. A. 100 (22) (2003) 12564-12569.

[45] C. D. Schwieters, J. J. Kuszewski, T. N, G. M. Clore, The xplor-nih nmr molecular structure determination package, J. Magn. Res. 160 (2003) 66-74.

[46] W. Humphrey, A. Dalke, K. Schulten, VMD: Visual molecular dynamics, J. Mol. Graph. 14 (1) (1996) 33.

[47] A. Damjanovic, I. Kosztin, U. Kleinekathfer, K. Schulten, Excitons in a photosynthetic light-harvesting system: a combined molecular dynamics, quantum chemistry, and polaron model study, Phys. Rev. E 65 (2002) 031919.

[48] L. Kale, R. Skeel, M. Bhandarkar, R. Brunner, A. Gursoy, N. Krawetz, J. Phillips, A. Shinozaki, K. Varadarajan, K. Schulten, Namd2: greater scalability for parallel molecular dynamics, J. Comput. Phys. 151 (1) (1999) $283-312$.

[49] A. D. MacKerell Jr, D. Bashford, M. Bellott, R. L. Dunbrack Jr, J. Evanseck, M. J. Field, S. Fischer, J. Gao, H. Guo, S. Ha, D. Joseph, L. Kuchnir, K. Kuczera, F. T. K. Lau, C. Mattos, S. Michnick, T. Ngo, D. T. Nguyen, B. Prodhom, I. W. E. Reiher, B. Roux, M. Schlenkrich, J. Smith, R. Stote, J. Straub, M. Watanabe, J. Wiorkiewicz-Kuczera, D. Yin, M. Karplus, Allhydrogen empirical potential for molecular modeling and dynamics studies of proteins using the charmm22 force field, J. Phys. Chem. B 102 (1998) 35863616.

[50] A. Pandit, R. Vischers, I. van Stokkum, R. Kraayenhof, R. van Grondelle, Oligomerization of Light-Harvesting I antenna peptides of Rhodospirillum rubrum, Biochemistry 40 (2001) 12913-12924. 
[51] A. Végh, B. Robert, Spectroscopic characterisation of a tetrameric subunit form of the core antenna protein from Rhodospirillum rubrum., FEBS Lett. 528 (2002) 222-226.

[52] A. Schubert, A. Stenstam, W. J. D. Beenken, J. L. Herek, R. Cogdell, T. Pullerits, V. Sundstrom, In vitro self-assembly of the light harvesting pigment-protein $\mathrm{lh} 2$ revealed by ultrafast spectroscopy and electron microscopy, Biophys. J. 86 (4) (2004) 2363-2373. 